\title{
Rotavirus infection beyond the gut
}

This article was published in the following Dove Press journal:

Infection and Drug Resistance

José Gómez-Rial, 1,2 Sonia

Sánchez-Batán, ${ }^{2}$ Irene

Rivero-Calle, ${ }^{1,3}$ Jacobo

Pardo-Seco,' José María

Martinón-Martínez,'

Antonio Salas, ${ }^{1,4,5}$ Federico

Martinón-Torres 1,3

'Grupo de Investigación en Genética, Vacunas, Infecciones y Pediatría

(GENVIP), Instituto de Investigaciones Sanitarias (IDIS), Hospital Clínico

Universitario de Santiago de

Compostela (SERGAS), Galicia,

Spain; 'Laboratorio de Inmunología,

Servicio de Análisis Clínicos, Hospital

Clínico Universitario de Santiago de

Compostela (SERGAS), Galicia, Spain;

${ }^{3}$ Translational Pediatrics and Infectious

Diseases, Department of Pediatrics,

Hospital Clínico Universitario de

Santiago de Compostela (SERGAS),

Galicia, Spain; ${ }^{4}$ Unidade de Xenética,

Departamento de Anatomía

Patolóxica e Ciencias Forense,

Instituto de Ciencias Forenses,

Facultade de Medicina, Universidade

de Santiago de Compostela, Galicia,

Spain; ${ }^{5}$ GenPoB Research Group,

Instituto de Investigaciones Sanitarias

(IDIS), Hospital Clínico Universitario

de Santiago de Compostela (SERGAS),

Galicia, Spain

Correspondence: Federico

Martinón-Torres

Grupo de Investigación en Genética,

vacunas, Infecciones y Pediatría

(GeNvlP), Instituto de Investigaciones

Sanitarias (IDIS), Hospital Clínico

Universitario de Santiago de

Compostela (SeRGAS), Travesa da

Choupana s/n 15706 Santiago de

Compostela, Galicia, Spain

Tel +3498I 955373

Fax +34 98I 950596

Email federico.martinon.torres@sergas.es

\begin{abstract}
The landscape of rotavirus (RV) infection has changed substantially in recent years. Autoimmune triggering has been added to clinical spectrum of this pathology, which is now known to be much broader than diarrhea. The impact of RV vaccines in these other conditions is becoming a growing field of research. The importance of host genetic background in RV susceptibility has been revealed, therefore increasing our understanding of vaccine effectiveness and giving some clues about the limited efficacy of RV vaccines in low-income settings. Also, interaction of RV with intestinal microbiota seems to play a key role in the process of infection vaccine effect. This article reviews current findings on the extraintestinal impact of $\mathrm{RV}$ infection and their widening clinical picture, and the recently described mechanisms of host susceptibility to infection and vaccine effectiveness. RV infection is a systemic disease with clinical and pathophysiological implications beyond the gut. We propose an "iceberg" model for this pathology with almost hidden clinical implications away from the gastrointestinal tract and eventually triggering the development of autoimmune diseases. Impact of current vaccines is being influenced by host genetics and gut microbiota interactions and these factors must be taken into account in the development of public health programs.
\end{abstract}

Keywords: rotavolution, extraintestinal, seizures, vaccines, autoimmunity

\section{Introduction}

For many years, rotavirus (RV) pathology has remained an undervalued condition and limited only to the gastrointestinal tract in the eyes of most clinicians. However, recent evidence from hidden systemic implications of RV infection has renewed interest in this pathology. ${ }^{1}$ It is now clear that RV goes beyond the gastrointestinal infection. The classic term "acute gastroenteritis (AGE) by RV" is increasingly replaced by "pathology by RV", reflecting the well-established systemic implications of the infection. ${ }^{2}$

This "rotavolution" - or change in the traditional clinical perception of RV infection - has been encouraged by the impact of RV vaccines, ${ }^{2}$ through a series of published unexpected benefits that have scrambled the long-held perception of diarrhea as the main or only clinical effect of $\mathrm{RV}^{3,4}$ In fact, diarrhea is not even necessary for the diagnosis of RV infection since an important percentage of rotaviremic patients show no clinical intestinal manifestations.

Furthermore, the recently established link between host genetics, gut microbiota and RV susceptibility have focused our interest on the interaction of RV with their host, and on the eventual "natural" resistance of some individuals to the infection or to the systemic spread of the virus. ${ }^{5}$ 
The present review aims to expand our understanding of this pathology, providing an updated rationale for the concept "rotavolution". 2,6 We propose an "iceberg" model for the RV pathology (Figure 1) that explains the underestimated or previously ignored clinical implications of this infection beyond the gastrointestinal tract.

\section{The clinical spectrum of RV}

The clinical status of RV infection is updated every year, with growing evidence pointing to a link between RV and the development of a number of autoimmune diseases in susceptible subjects as the most impactful systemic consequence. The RV pathology is systemic; the RV goes beyond the intestinal lumen irrespective of the presence or not of diarrhea. ${ }^{1}$ Since RV antigenemia detection is not a routine diagnostic tool, the impact of RV as a pathogen in children is underestimated, especially in the absence of diarrhea, where the routine RV diagnostic work-up is not even used. Seizures may constitute the most frequently recognized extraintestinal manifestation of RV infection. ${ }^{7}$

\section{Acute extraintestinal manifestations}

The role of RV infection as the cause of seizure or clinical neurological illness is well established in the scientific lit- erature $;{ }^{8}$ these are the most common neurological symptoms with an incidence of $4.0 \%$ to $7.7 \%$ of patients, respectively. ${ }^{7}$ Furthermore, several reports described diffuse cerebral white matter lesions in neonates with RV-associated seizures. ${ }^{9}$ The pathophysiological mechanism of RV-induced seizure remains elusive. A hypothesis has been proposed that argues a key role for the viral nonstructural protein 4 (NSP4) through a disruption of $\mathrm{Ca}^{2+}$ homeostasis that may result in neurotoxicity and neurotransmitter dysregulation. ${ }^{10}$ NSP4 has been demonstrated to act as an enterotoxin-inducing secretion of $\mathrm{Cl}^{-}$ions and water through phospholipase-dependent elevation of cytosolic $\mathrm{Ca}^{2+}$. However, this effect was not limited to the intestinal cells as NSP4 can bind to the surface of various cell types through interaction with glycosaminoglycans. ${ }^{11}$ Thus, the pathophysiological effects of NSP4 may have a broader cellular tropism and exert a wider range of physiological effects in the host. NSP4 has also been shown to have inherent membrane destabilizing properties. ${ }^{12}$ In this vein (see also below), it has been proposed that changes in NSP4 susceptibility may affect the impact of RV vaccines in seizures. ${ }^{13}$

Another possible explanation for the RV-induced seizure is through direct central nervous system (CNS) infection

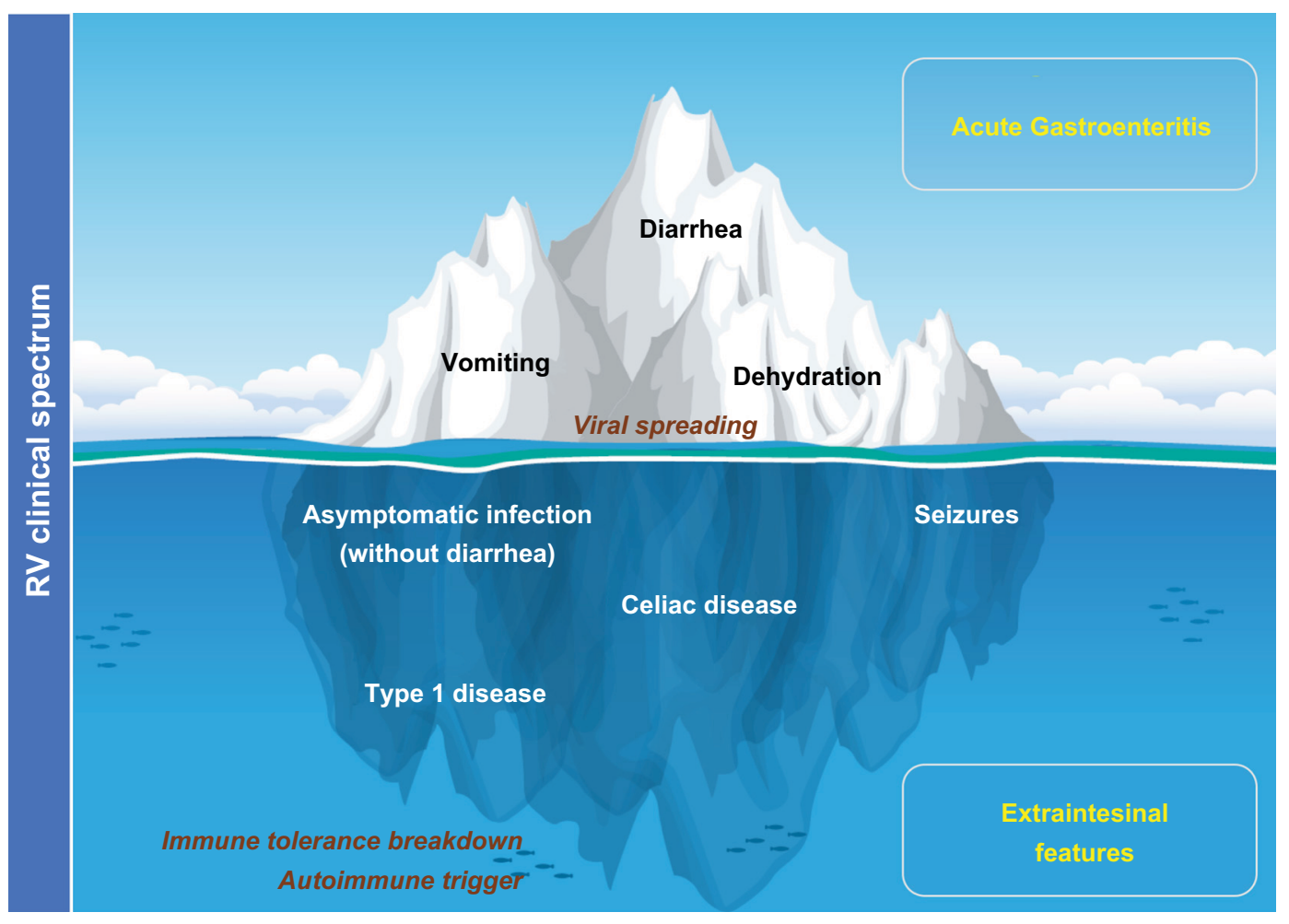

Figure I The "iceberg" model of RV infection proposal: AGE and diarrhea are just the most obvious and frequent clinical picture of the pathology by RV.

Notes: Systemic viral spreading occurs and might produce several other extraintestinal manifestations such as seizures in the CNS (Table I). Moreover, RV infection may be a trigger for the development of autoimmune pathology in individuals with a specific genetic background through a proposed mechanism of immune tolerance breakdown at early ages.

Abbreviations: AGE, acute gastroenteritis; CNS, central nervous system; RV, rotavirus. 
action. ${ }^{14}$ This hypothesis is supported by several studies demonstrating RV detection on spinal fluid, ${ }^{15}$ and by experimental animal models. ${ }^{16}$ However, RV evidence has not been sought in spinal fluid in all cases nor the pathogenic mechanism established.

Other possible acute extraintestinal manifestations are listed in Table 1; these are mainly based on case reports and therefore it is more difficult to estimate their real burden.

\section{Clinical significance of antigenemia}

$\mathrm{RV}$ antigenemia and ribonucleic acid (RNAemia) detection are common findings in RV infection ${ }^{17,18}$ even in the absence of diarrhea: ${ }^{16}$ furthermore, these features have recently been linked to increased severity of fever and vomiting by an unknown mechanism, ${ }^{19}$ and particularly associated to the RV genotype G1P[8] infections. However, no correlation between $\mathrm{RV}$ viremia or antigenemia and diarrhea has been found.

Previously, the activation of dendritic cells in the acute phase of infection appeared to correlate with levels of antigenemia, and a high prevalence of NSP4 gene was detected in peripheral blood mononuclear cells, suggesting white blood cells as the source of extraintestinal viral replication. ${ }^{20}$ Furthermore, a correlation between cytokine levels and RV antigenemia was found in patients with fever, suggesting that the severity of systemic infection contributes to the systemic manifestation of disease. ${ }^{21}$

\section{Trigger for autoimmune diseases}

The role of RV as an environmental trigger of several autoimmune diseases has been the focus of interest in the last few

Table I Extraintestinal manifestations of RV infection.

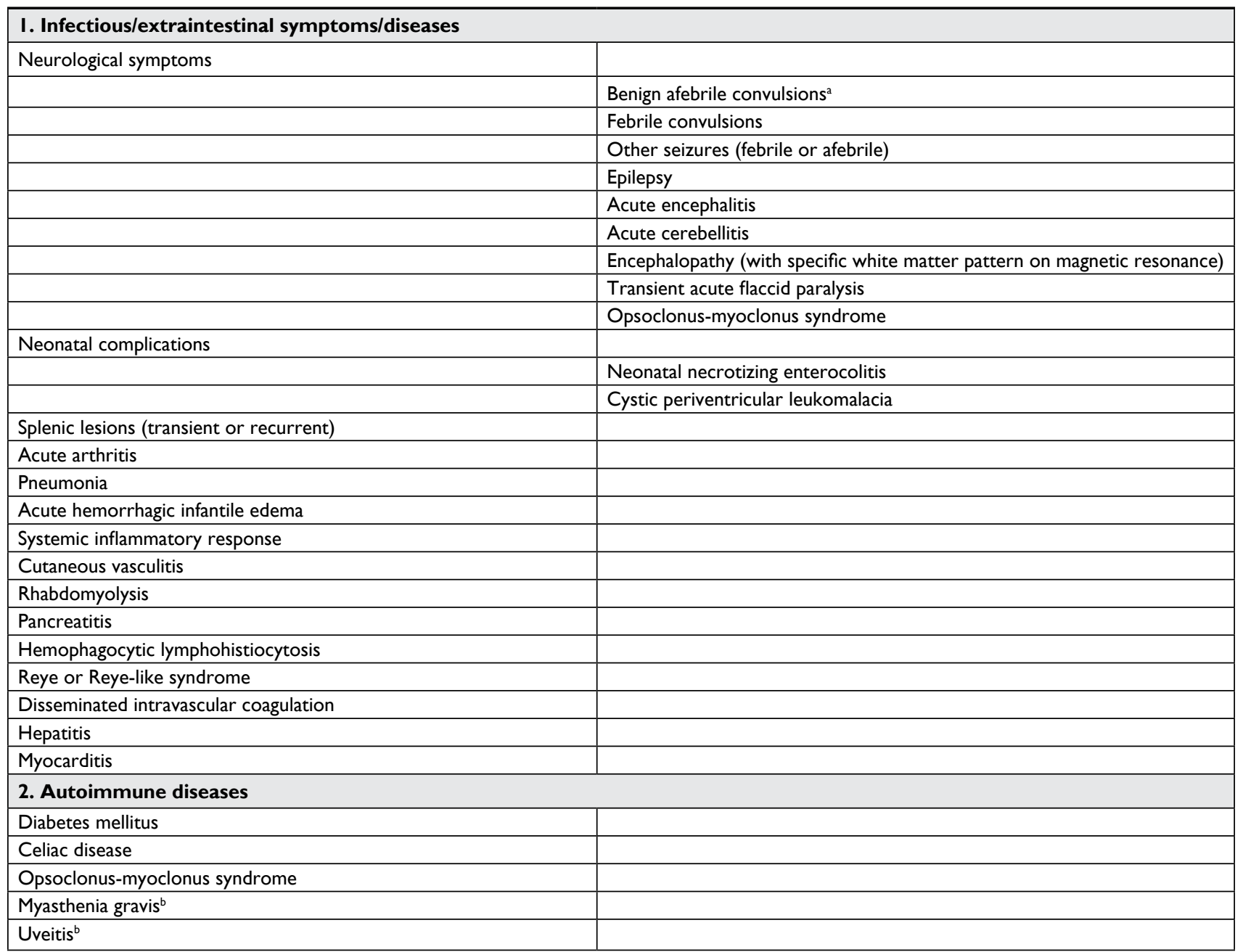

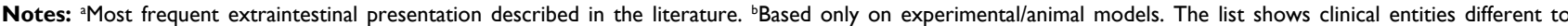
diarrhea that have been reported or suggested to be related to RV infection, (I) or triggered by RV infection in susceptible subjects (2). These symptoms can be present alone or in combination, with or without diarrhea. See text for further explanations. Adapted from J Infect, 72 Suppl, Rivero-Calle I, Gómez-Rial J, Martinón-Torres F, Systemic features of rotavirus infection, S98-S105, copyright 2016, with permission from Elsevier. ${ }^{2}$

Abbreviation: RV, rotavirus. 
years. $^{22}$ Special attention has been paid to celiac disease, an autoimmune enteropathy, where a high frequency of RV infections may increase the risk of celiac disease in childhood in genetically predisposed individuals. ${ }^{23}$ Frequent RV infections during infancy predicted a higher risk of celiac disease in childhood with a relative risk of 3.76 for individuals with two or more infections. ${ }^{23}$ A study carried out in Italian patients demonstrated that children born in the summer were at higher risk to develop celiac disease than subjects born in other seasons; this study pointed to the coincidence in the timing of the first introduction of gluten and the highest peak of RV infection as possible causes. ${ }^{24}$

In a recent study Kemppainen et $\mathrm{al}^{25}$ showed that this risk was modified by human leukocyte antigen (HLA) genotype, gluten consumption, breastfeeding and also RV vaccination, indicating complex interactions among infections, genetics, and diet in the development of celiac disease.

The mechanism for this association is unclear. Several authors have proposed a hypothesis of molecular mimicry between RV capsid protein VP7 and the human-tissue transglutaminase, the main autoantigen of the celiac disease. ${ }^{26}$ It has been shown that VP7 can be recognized by certain antitransglutaminase antibodies present in the serum of celiac patients; moreover, these antibodies are present before the onset of the celiac disease, preceding the detection of antitransglutaminase and anti-endomysium antibodies. However, Ziberna et $\mathrm{al}^{27}$ have recently questioned this hypothesis in a study that showed lack of evidence for this RV-dependent molecular mimicry as a trigger for celiac disease.

Lastly, another interesting mechanism has been proposed by Bouziat et $\mathrm{al}^{28}$ supporting a role for infection with reovirus (the double-stranded RNA virus family to which the RV belongs to) in triggering the development of celiac disease. Using a viral infection model, the authors showed that reovirus infection disrupts intestinal immune homeostasis at inductive and effector sites of oral tolerance, by suppressing peripheral regulatory cells conversion and promoting an exacerbated immune response to dietary antigens, in a type one interferon (IFN)-related pathway. ${ }^{28}$

Similarly, RV infection has been claimed as a triggering factor for type I diabetes mellitus, an autoimmune endocrinopathy leading to selective destruction of insulin-producing pancreatic beta cells. ${ }^{29}$ Data from experimental animals as well as in vitro studies indicate that RV, like other viruses, is clearly able to modulate the development of diabetes via different mechanisms, including direct-beta cells lysis, bystander activation of autoreactive $T$ cells, suppression of regulatory cells, and molecular mimicry. ${ }^{30,31}$ However, the exact mechanism is not entirely clear and some authors consider this association unlikely. ${ }^{32}$

Using a bioinformatics approach, RV VP6 protein has also been identified as a potential threat for myasthenia gravis, a chronic muscular neurodegenerative autoimmune disorder. ${ }^{33}$ In this in silico study, most conserved structural protein VP6 matches at two regions with ryanodine receptor, the autoimmune target associated with the myasthenia. Furthermore, it was observed that these regions remain conserved in all circulating RV strains and showed significant antigenicity with respect to myasthenia-associated HLA haplotypes.

\section{The lessons learnt from RV vaccination}

Implementation of RV vaccines has substantially decreased hospitalizations from RV and all-cause AGE among children $<5$ years of age. ${ }^{34}$ Vaccination has also had an indirect effect among unvaccinated older children and young adults. ${ }^{35}$ More surprisingly, the introduction of RV vaccine has also impacted on extraintestinal RV manifestations in a way that we are just beginning to understand.

\section{Impact of RV vaccine on seizures}

Payne et $\mathrm{al}^{4}$ were the first to demonstrate that a full course of RV vaccination significantly reduced the risk of childhood seizures during the year following vaccination, with an $18-21 \%$ decrease in relative risk of seizures requiring hospitalization as compared with children not receiving the vaccine. Several other teams have found similar protective effects for RV vaccine against seizures and convulsions, ${ }^{13,36}$ even in a moderate vaccine coverage scenario (Figure 2). The unexpected benefit of RV vaccination in these studies seems more marked in the youngest infants ( $<5$ years). Yeom et $\mathrm{al}^{13}$ described changes in the clinical characteristics of RVassociated seizures after the introduction of RV vaccines, with more common afebrile seizures and a longer interval between gastroenteritis symptoms and the onset of seizures. Action on NSP4 is identified as the cause of this altered clinical course, related to changes in NSP4 immunity and the generation of anti-NSP4 IgG antibodies after vaccination. ${ }^{37}$

In contrast, a recent ecological study carried out in Spanish (Valencia; southeast Spain) children $<5$ years old by Orrico-Sánchez et $\mathrm{al}^{38}$ has reported a lack of impact of $\mathrm{RV}$ vaccine on seizure hospitalization rates. There are some issues in the study by Orrico-Sánchez et $\mathrm{al}^{38}$ that might help to explain the differences with all the other studies (Figure 2 ). For instance, the authors included primary care patients and used absolute figures instead of rates. In addition, these 


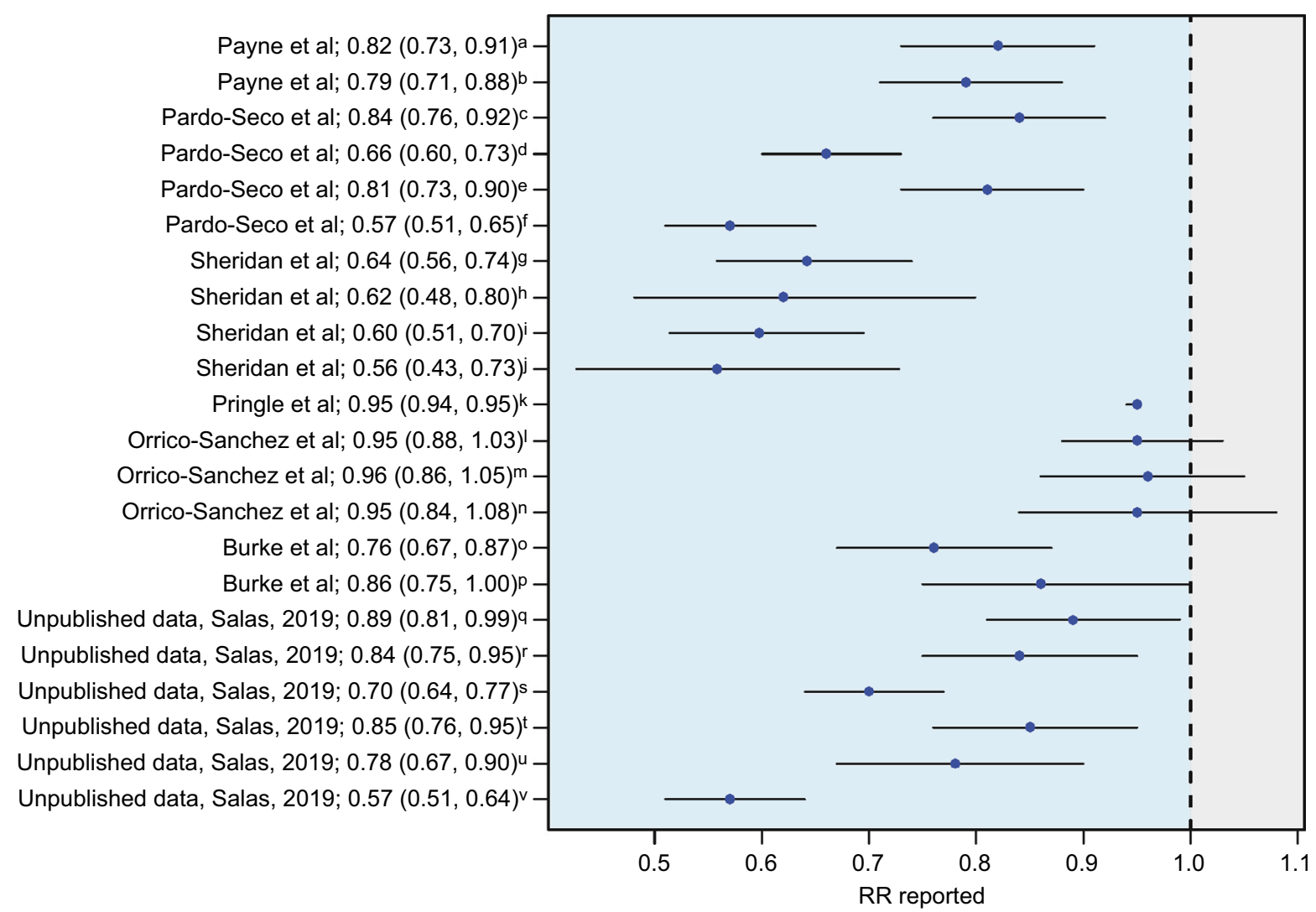

Figure 2 Data from different studies indicating the relation existing between RV vaccination coverage and seizures/convulsions expressed as RRs and their $95 \% \mathrm{Cl}$. Notes: RR values below one suggest a heterologous effect or an unforeseen direct effect of the RV vaccine that would favor a lower incidence of seizures/convulsions in

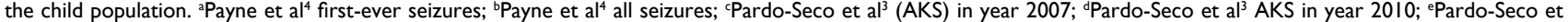

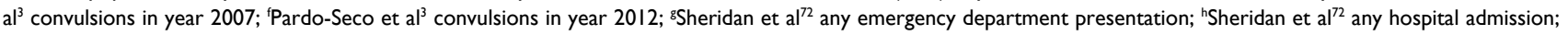

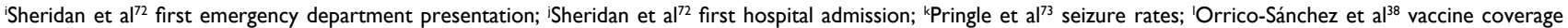
$1 \%-19 \%$; ${ }^{\mathrm{m}}$ Orrico-Sánchez et al ${ }^{38}$ vaccine coverage $20 \%-39 \%$; ${ }^{\mathrm{O}}$ Orrico-Sánchez et al ${ }^{38}$ vaccine coverage $>39$; ${ }^{\circ}$ Burke et al ${ }^{74}$ full vaccination; ${ }^{\mathrm{P} B u r k e}$ et al $\mathrm{I}^{74}$ partially vaccinated; Unpublished data AKS and RV vaccination coverage 1\%-14\%; Unpublished data AKS and RV vaccination coverage 15\%-29\%; Unpublished data AKS and RV vaccination coverage $>29 \%$; Unpublished data convulsions and RV vaccination coverage 1\%-14\%; Unpublished data convulsions and RV vaccination coverage I5\%-29\%; Unpublished data convulsions and RV vaccination coverage $>29 \%$.

Abbreviations: AKS, all kinds of seizures; RV, rotavirus.

authors used a mixed Poisson regression model involving multiple variables (including vaccination coverage) to avoid confounder effects. However, this model includes variables such as time since vaccine introduction, which could be highly correlated with vaccination coverage (as time goes by, the vaccination coverage should increase), which can result in overfitting and statistical noise. Despite this the study states no impact of RV vaccine on seizures hospitalization, it is remarkable that the trend shown by their data indicates a relative risk in the same direction as the studies with positive findings. ${ }^{38}$ More recently, Biggart et a ${ }^{39}$ have also published a lack of effect of the monovalent $\mathrm{RV}$ vaccine on childhood seizure hospitalizations in the UK using an interrupted time series analysis.

If there is a beneficial impact on seizures and whether there is a potential benefit exerted by RV vaccines mainly due to the prevention of RV infections in infants otherwise susceptible to the neurological tropism of RV, and/or a true heterologous effect of the vaccine, remains unknown. ${ }^{2}$ Indeed, now more than ever, more studies are needed to clarify the effect of RV vaccines on seizures.

\section{Impact of RV vaccines on autoimmune manifestations}

Vaarala et $\mathrm{a}^{40}$ reported that $\mathrm{RV}$ vaccination did not alter the risk of celiac disease and type I diabetes. In contrast, the recent study by Kemppainen et $\mathrm{a}^{25}$ proposed a protective association between RV vaccine and the development of celiac disease, considering RV as an important environmental factor for triggering autoimmunity. This study showed a reduced risk of celiac disease autoimmunity in children vaccinated against RV who had been introduced to gluten 
before 6 months of age, with a HR of 0.57 . More studies are needed to clarify the effects of RV vaccines on autoimmune manifestations.

\section{Molecular mechanisms involved in the systemic interaction of RV}

The underlying molecular mechanism of RV attachment and entry into host is now well established (Figure 3). Trypsinlike proteases from the host intestinal lumen cleave the VP4 capsid protein to produce an N-terminal VP8* and the C-terminal VP5* peptides. This proteolytic processing of the outer viral spike VP4 seems essential for infectivity, ${ }^{41}$ leading to a more stable, rigid spike structure and displaying the distal lectin domain of VP8* molecule for receptor binding and attachment to the mucosal epithelia. ${ }^{42}$

The ligands for VP8* in host epithelia have been identified as oligosaccharides of the histo-blood group antigens (HBGAs) family, present in mucosal secretion, epithelial intestinal, and red blood cells. ${ }^{43}$ All of these HBGAs have in common an $\alpha-1,2$-fucose linked residue, added by the $\alpha$-1,2-fucosyltransferase enzyme, encoded by the FUT2 gene. FUT2-null homozygotes determine the non-secretor phenotype; the evidence suggesting that homozygote carriers develop a natural resistance to RV infection. Thus, FUT2 expression seems to be relevant for viral infectivity and serves as a marker for host susceptibility.

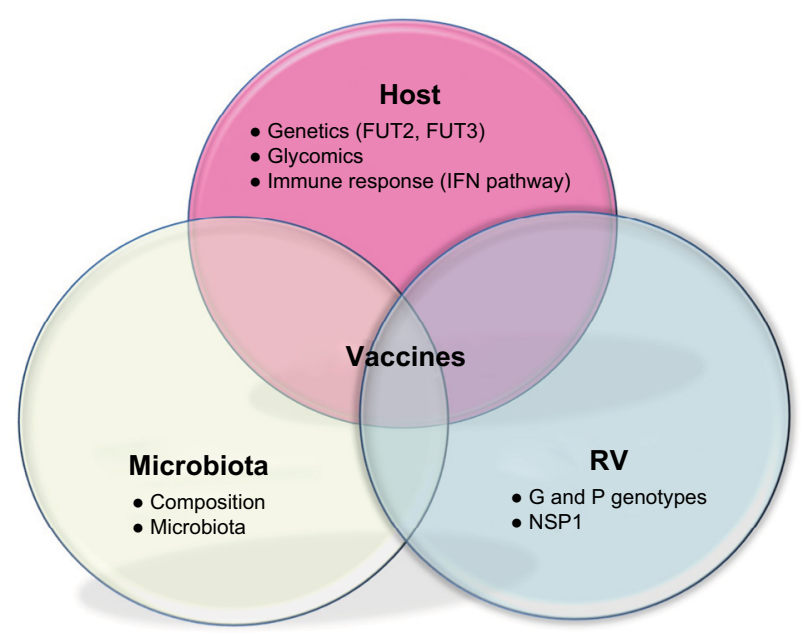

Figure 3 Interactions in RV pathology: host, microbiota and RV.

Notes: Host background includes genetics through expression of histo-blood groups in epithelia, glycomics with the importance of sugars for RV interaction with host cells, and the IFN pathway, key in immune host defense. Intestinal microbiota composition and metagenome influence the course of RV infection through interactions between commensal microbial flora and pathogen. RV proteins (VP4 and VP7) determined by G and P genotypes and enterotoxin NSPI play an important role in RV attachment, cell internalization and pathogeny. Overall, effectiveness of $R V$ vaccines may be influenced by all these elements.

Abbreviations: IFN, interferon; RV, rotavirus; NSPI, nonstructural protein I.
However, the mechanism for viral dissemination from the gut is not clear. A neonatal mouse model of RV infection suggested that extraintestinal spread occurs via a lymphatic pathway, is primarily determined by non-structural protein NSP3, and can be modified by the VP6 capsid protein. ${ }^{44}$ Cells from the lymphocytic or myeloid lineage were proposed as viral replication sites during the extraintestinal spread. ${ }^{20}$

RV tropism toward neuronal cells could in some way explain why the CNS is the main focus of extraintestinal affectation, and the viral dissemination mechanism could involve the attachment to HBGAs or other specific cell receptors. A mechanism of retrograde axon transport has also been proposed, whereby synaptic vesicles returned to the CNS from axon terminals, as described for other pathogens (herpesvirus, rabies, polio virus). ${ }^{45}$

Overall, it seems that viral spread depends on viral factors (NSP3, VP6), but other components of the process might also be related to genetic host. Recently, a blood-whole transcriptome analysis has revealed that the host downregulates glycophorin expression in a suggested mechanism of viral spread inhibition. ${ }^{46}$ Glycophorins are cell-membrane glycoproteins rich in sialic acid, a monosaccharide often associated in the literature with RV interaction. ${ }^{47}$ The role of this mechanism in avoiding systemic spread remains to be clarified.

\section{The role of the host in $R V$ infection Host genetic component in RV infection}

It has been proposed that the HBGAs of the host play an important role in RV cell attachment. ${ }^{48}$ These HBGAs contain a carbohydrate structure, namely $\mathrm{H}$ type I antigen, whose synthesis is dependent on the FUT2 gene expression, which determines the secretor status. There is abundant evidence in the literature suggesting a strong association between the FUT2 gene and risk of infection with RV and other enteropathogens such as norovirus. ${ }^{5,49,50}$ Individuals with secretor phenotype have an increased susceptibility to RV, especially to $\mathrm{P}[8]$ genotype; conversely, severe RV gastroenteritis is virtually absent among children without $F U T 2$ expression in the intestinal epithelium. ${ }^{51}$ In addition, FUT3 - also related to HBGA expression and determinant of the Lewis antigen - has been proposed as a potential determinant of host susceptibility to $\mathrm{RV}^{49}$ The recent meta-analysis by Bustamante et $\mathrm{al}^{50}$ points to the single-nucleotide polymorphism rs601338 (W154X) in the FUT2 gene as the causal variant in diarrhea at 1 year of age. The A-allele at this position results in a truncated protein and a lower risk of diarrhea caused by RV and norovirus. ${ }^{50}$

The host susceptibility to RV infection mediated by FUT2 and FUT3 is RV genotype dependent. ${ }^{49} \mathrm{RV} \mathrm{P}[8]$ infects exclu- 
sively Lewis- and RV secretor-positive children, in contrast to RV P[6] strains that infect mainly Lewis-negative children, regardless of their secretor FUT2 status.

In view of these findings, differences in host genetic susceptibility could have implications in vaccine efficacy and management. The $\mathrm{P}[8]$ genotype is the main component of the two licensed RV vaccines, therefore the proportion of Lewis-negative individuals must be taken into account in order to assess the vaccine efficacy. Thus, for instance, it is reasonable to speculate about a lower vaccine efficacy in Lewis-negative individuals ${ }^{49}$ evidence in this direction has been recently provided by Bucardo et al by analyzing Nicaraguan children. ${ }^{52}$ In particular, the predominance of Lewis-negative phenotype among African populations is worth noting, ${ }^{53}$ as these population differences in HBGA expression may be responsible for discrepancies in the vaccine protection detected for the current $\mathrm{RV}$ vaccines in low-income vs high-income settings. ${ }^{54}$

Additionally, it was proposed that neonatal resistance to the $\mathrm{P}[8]$ and $\mathrm{P}[4]$ genotypes could be explained by the absence of Lewis antigen on the cell surface, as young children are usually Lewis-negative until 1-2 months of age. ${ }^{55}$ Thus, neonatal children might be susceptible to RV P[6] genotypes only, and not to $\mathrm{P}[8]$ and $\mathrm{P}[4]$ genotypes.

\section{The host microbiota perspective}

New evidence points to the host gut microbiota as a key player necessary for a viral pathogen to cause infection. ${ }^{56}$ Data from experimental studies demonstrated that the use of germ-free animals or antibiotic treatments results in a reduced rate of $\mathrm{RV}$ infection. ${ }^{57}$ These findings highlight the importance of the presence of certain bacterial types in the gut microbiota for RV attachment and infection. This microbiome composition is also related to HBGA and host genetics. ${ }^{5}$ The intestinal microbiota itself affects host intestinal glycosylation patterns and mucin production, including fucosylation of HBGA. ${ }^{58}$ The relationship between gastrointestinal viruses and commensal bacteria remains to be elucidated, although there is growing evidence indicating that RV susceptibility and infectivity must be understood within an integrated framework, whereby host genetic and gut microbiota factors cannot be separated.

Alternatively, HBGA, host genetic and microbiota interactions may be also modulating vaccine strain replication. The composition of the bacterial microbiota may shape the response to $\mathrm{RV}$ vaccines, ${ }^{59}$ and this may contribute to their low efficacy in low-income settings. In a study conducted in Pakistani population, Harris et $\mathrm{al}^{6}$ have recently argued that RV vaccine response correlates with the infant gut microbiota composition. The response to the monovalent RV vaccine (RV1) correlated with a higher relative abundance of Clostridium and Proteobacteria, including Serratia and Escherichia coli. ${ }^{6}$ Therefore, identification of key bacteria that correlate with RV vaccine efficacy could be important for designing future interventions in low-efficacy vaccine settings.

Conversely, RV vaccination appears to be inconsequential for the process of individual microbiome establishment, as recently demonstrated. ${ }^{60}$ Accordingly, it seems that microbial colonization of the intestine occurs during the first months of life, and oral RV vaccination does not show any major effect upon the infant gut microbiota.

\section{The immunological perspective}

The mechanisms responsible for immunity to RV in humans are not completely understood but it seems clear that immunological factors are crucial in susceptibility to RV infection and systemic spread. RV infects primarily enterocytes, and the virus is detected by cytoplasmic pattern recognition receptors (retinoic acid-induced gene-1, also known as RIG-I, and IFN-induced helicase $\mathrm{C}$ domain-containing protein 1, also known as MDA5). These immune receptors recognize viral $\mathrm{RNA}^{61}$ and induce type I and type III IFN responses $^{62}$ in host. At this point, a real battle for controlling the IFN machinery occurs between RV and the host. ${ }^{63}$ The nonstructural RV protein (NSP1) downregulates IFN expression, inducing degradation of multiple members of the family of the IFN regulatory factors, ${ }^{64}$ which might underlie the poor innate immune response to RV in the natural infection. ${ }^{65}$ In addition, toll-like receptor 3 , another pattern recognition receptor, has been recently associated to the age-dependent resistance to RV disease in experimental animal models, as both proteins are expressed at higher levels in adult animals. ${ }^{66}$

A combination of two innate cytokines, IL-18 and IL-22, has been proposed as a key mediator in the clearance of RV by the innate immune system in mice. ${ }^{67}$ However, RV infection evades the innate immune system efficiently, indicating that the mediators of RV clearance might be cells of the adaptive immune system, as $\mathrm{CD}^{+}$cytotoxic $\mathrm{T}$ cells that can be detected in the blood of children with RV disease. ${ }^{68}$ However, some authors consider that circulating RV-specific $\mathrm{CD}^{+} \mathrm{T}$ cells have a poor functional profile and are B cells and antibodies primary determinant in clearance of primary infection and absolutely necessary for development of immunity against reinfection. ${ }^{69}$ 
Data from mouse experimental models indicate that immunological effectors responsible for clearance of RV from blood and from intestine are similar, but it is unknown if these effectors are induced solely in blood or intestine or at both sites. Furthermore, recent studies have shown that polymorphisms in genes encoding factors of the immune system can influence the host response to infection and the course of disease in RV and other viral infections. ${ }^{70}$ Data from animal models showed that IFN- $\lambda$ genetic polymorphisms affected host control of RV infection; ${ }^{71}$ thus, genetic variation of key immune mediators could potentially influence the course of the disease and determine the degree of viral spread.

Accordingly, deeper knowledge of the immune mechanisms elicited in RV infection, especially at mucosa level, is necessary in order to predict the potential influence of immune mediator genetic variations on the course and evolution of infection. Similarly, to understand the "protective" effect of RV vaccines, we might hypothesize that the factor responsible for this protective association might be the prevention of the exacerbated inflammatory response elicited by the natural infection with the subsequent break of immune tolerance, as opposed to a more controlled immune response of vaccination. Overall, more research is warranted to elucidate the eventual impact of RV vaccines on autoimmune response.

\section{Conclusion}

We are witnessing an authentic "rotavolution" in the understanding of RV pathology. RV infection consequences might be described as an "iceberg" model whereby diarrhea is the most visible tip (Figure 1). Autoimmune triggering through RV infection constitutes an interesting mechanism for certain diseases; if confirmed, this finding points to new ways of intervention in these diseases. The role of current $\mathrm{RV}$ vaccines on these widened clinical spectra remains to be elucidated, and it may constitute a possible heterologous effect, an unforeseen direct effect, or a combination of both. In addition, host genetic background and gut microbiota are being revealed as key influential factors for RV infection and for vaccine effectiveness. The design of new vaccines and public health programs would benefit from taking all these interactions into account. It is now known that the causes of infectious diseases are genetically complex and multifactorial, involving complex interactions between the host and pathogen factors. High-throughput "-omic" strategies (genomics, transcriptomics, metabolomics, glycomics, vaccinomics, etc) are now beginning to revolutionize the way we understand mechanisms of viral infection. Application of these strategies to the rotavolution era will shed new and necessary light on the RV iceberg model.

\section{Author contributions}

All authors contributed to data analysis, drafting or revising the article, gave final approval of the version to be published, and agree to be accountable for all aspects of the work

\section{Disclosure}

This work was supported by grants from the Instituto de Salud Carlos III (Proyecto de Investigación en Salud, Acción Estratégica en Salud): project GePEM ISCIII/PI16/01478/ Cofinanciado FEDER (AS) and project ReSVinext ISCIII/ PI16/01569/Cofinanciado FEDER (FMT); Consellería de Sanidade, Xunta de Galicia (RHI07/2-intensificación actividad investigadora, PS09749 and 10PXIB918184PR), Instituto de Salud Carlos III (Intensificación de la actividad investigadora 2007-2012, PI16/01569), Fondo de Investigación Sanitaria (FIS; PI070069/PI1000540) del plan nacional de I + D + I (FMT), and 2016-PG071 Consolidación e Estructuración REDES 2016GI-1344 G3VIP (Grupo Gallego de Genética Vacunas Infecciones y Pediatría, ED341D R2016/021) (AS and FMT). FMT has received honoraria from GSK, Pfizer, Sanofi Pasteur, MSD, and Janssen for taking part in advisory boards and expert meetings, and for acting as speaker in congresses outside the scope of the submitted work. FMT has also acted as principal investigator in RCTs of the above-mentioned companies as well as Seqirus, Ablynx, Regeneron, Abbot, Novavax and Medimmune, with honorarium paid to his institution. The authors report no other conflicts of interest in this work.

\section{References}

1. Blutt SE, Kirkwood CD, Parreño V, et al. Rotavirus antigenaemia and viraemia: a common event? Lancet. 2003;362(9394):1445-1449.

2. Rivero-Calle I, Gómez-Rial J, Martinón-Torres F. Systemic features of rotavirus infection. J Infect. 2016;72 Suppl:S98-S105.

3. Pardo-Seco J, Cebey-López M, Martinón-Torres N, et al. Impact of rotavirus vaccination on childhood hospitalization for seizures. Pediatr Infect Dis J. 2015;34(7):769-773.

4. Payne DC, Baggs J, Zerr DM, et al. Protective association between rotavirus vaccination and childhood seizures in the year following vaccination in US children. Clin Infect Dis. 2014;58(2):173-177.

5. Payne DC, Currier RL, Staat MA, et al. Epidemiologic association between FUT2 secretor status and severe rotavirus gastroenteritis in children in the United States. JAMA Pediatr. 2015;169(11):1040-1045.

6. Harris V, Ali A, Fuentes S, et al. Rotavirus vaccine response correlates with the infant gut microbiota composition in Pakistan. Gut Microbes. 2018;9(2):93-101.

7. Lloyd MB, Lloyd JC, Gesteland PH, Bale JF. Rotavirus gastroenteritis and seizures in young children. Pediatr Neurol. 2010;42(6):404- 408. 
8. Difazio MP, Braun L, Freedman S, Hickey P. Rotavirus-induced seizures in childhood. J Child Neurol. 2007;22(12):1367-1370.

9. Lee KY, Weon YC, Choi SH, Oh KW, Park H. Neurodevelopmental outcomes in newborns with neonatal seizures caused by rotavirusassociated leukoencephalopathy. Seizure. 2018;56:14-19.

10. Yeom JS, Kim YS, Park JS, et al. Role of Ca2+ homeostasis disruption in rotavirus-associated seizures. J Child Neurol. 2014;29(3):331-335.

11. Didsbury A, Wang C, Verdon D, Sewell MA, McIntosh JD, Taylor JA. Rotavirus NSP4 is secreted from infected cells as an oligomeric lipoprotein and binds to glycosaminoglycans on the surface of non-infected cells. Virol J. 2011;8:551.

12. Tian P, Ball JM, Zeng CQ, Estes MK. The rotavirus nonstructural glycoprotein NSP4 possesses membrane destabilization activity. $J$ Virol. 1996;70(10):6973-6981.

13. Yeom JS, Kim YS, Kim RB, et al. Impact of rotavirus vaccine introduction on rotavirus-associated seizures and a related possible mechanism. J Child Neurol. 2015;30(6):729-734.

14. Goldwater PN, Rowland K, Thesinger M, et al. Rotavirus encephalopathy: pathogenesis reviewed. J Paediatr Child Health. 2001;37(2):206-209.

15. Nishimura $S$, Ushijima $H$, Nishimura $S$, et al. Detection of rotavirus in cerebrospinal fluid and blood of patients with convulsions and gastroenteritis by means of the reverse transcription polymerase chain reaction. Brain Dev. 1993;15(6):457-459.

16. Crawford SE, Patel DG, Cheng E, et al. Rotavirus viremia and extraintestinal viral infection in the neonatal rat model. $J$ Virol. 2006;80(10):4820-4832.

17. Blutt SE, Matson DO, Crawford SE, et al. Rotavirus antigenemia in children is associated with viremia. PLoS Med. 2007;4(4):e121.

18. Ramani S, Paul A, Saravanabavan A, et al. Rotavirus antigenemia in Indian children with rotavirus gastroenteritis and asymptomatic infections. Clin Infect Dis. 2010;51(11):1284-1289.

19. Hemming M, Huhti L, Räsänen S, Salminen M, Vesikari T. Rotavirus antigenemia in children is associated with more severe clinical manifestations of acute gastroenteritis. Pediatr Infect Dis J. 2014;33(4):366-371.

20. Moon S, Wang Y, Dennehy P, Simonsen KA, Zhang J, Jiang B. Antigenemia, RNAemia, and innate immunity in children with acute rotavirus diarrhea. FEMS Immunol Med Microbiol. 2012;64(3):382-391.

21. Sugata K, Taniguchi K, Yui A, et al. Analysis of rotavirus antigenemia and extraintestinal manifestations in children with rotavirus gastroenteritis. Pediatrics. 2008;122(2):392-397.

22. Vojdani A. A potential link between environmental triggers and autoimmunity. Autoimmune Dis. 2014;2014:437231.

23. Stene LC, Honeyman MC, Hoffenberg EJ, et al. Rotavirus infection frequency and risk of celiac disease autoimmunity in early childhood: a longitudinal study. Am J Gastroenterol. 2006;101(10):2333-2340.

24. Capriati T, Francavilla R, Castellaneta S, Ferretti F, Diamanti A. Impact of the birth's season on the development of celiac disease in Italy. Eur J Pediatr. 2015;174(12):1657-1663.

25. Kemppainen KM, Lynch KF, Liu E, et al; TEDDY Study Group. Factors that increase risk of celiac disease autoimmunity after a gastrointestinal infection in early life. Clin Gastroenterol Hepatol. 2017;15702(5):694 702.e5.

26. Dolcino M, Zanoni G, Bason C, et al. A subset of anti-rotavirus antibodies directed against the viral protein VP7 predicts the onset of celiac disease and induces typical features of the disease in the intestinal epithelial cell line T84. Immunol Res. 2013;56(2-3):465-476.

27. Ziberna F, De Lorenzo G, Schiavon V, et al. Lack of evidence of rotavirus-dependent molecular mimicry as a trigger of coeliac disease. Clin Exp Immunol. 2016;186(3):356-363.

28. Bouziat R, Hinterleitner R, Brown JJ, et al. Reovirus infection triggers inflammatory responses to dietary antigens and development of celiac disease. Science. 2017;356(6333):44-50.

29. Honeyman MC, Coulson BS, Stone NL, et al. Association between rotavirus infection and pancreatic islet autoimmunity in children at risk of developing type 1 diabetes. Diabetes. 2000;49(8):1319-1324.
30. van der Werf N, Kroese FG, Rozing J, Hillebrands JL. Viral infections as potential triggers of type 1 diabetes. Diabetes Metab Res Rev. 2007;23(3):169-183.

31. Pane JA, Fleming FE, Graham KL, Thomas HE, Kay TW, Coulson BS. Rotavirus acceleration of type 1 diabetes in non-obese diabetic mice depends on type I interferon signalling. Sci Rep. 2016;6:29697.

32. Blomqvist M, Juhela S, Erkkila S, et al. Rotavirus infections and development of diabetes-associated autoantibodies during the first 2 years of life. Clin Exp Immunol. 2002;128(3):511-515.

33. Sarkar T, Das S, Nandy P, Bhowmick R, Nandy A. In silico study of potential autoimmune threats from rotavirus infection. Comput Biol Chem. 2014;51:51-56.

34. Jonesteller CL, Burnett E, Yen C, Tate JE, Parashar UD. Effectiveness of rotavirus vaccination: a systematic review of the first decade of global postlicensure data, 2006-2016. Clin Infect Dis. 2017;65(5):840-850.

35. Lopman BA, Curns AT, Yen C, Parashar UD. Infant rotavirus vaccination may provide indirect protection to older children and adults in the United States. J Infect Dis. 2011;204(7):980-986.

36. Park SH, Kim YO, Kim HK, et al. Incidence of benign convulsions with mild gastroenteritis after introduction of rotavirus vaccine. Brain Dev. 2015;37(6):625-630.

37. Yeom JS, Kim YS, Jun JS, et al. NSP4 antibody levels in rotavirus gastroenteritis patients with seizures. Eur J Paediatr Neurol. 2017;21(2):367-373.

38. Orrico-Sánchez A, López-Lacort M, Muñoz-Quiles C, Díez-Domingo J. Lack of impact of rotavirus vaccines on seizure-related hospitalizations in children under 5 years old in Spain. Hum Vaccin Immunother. 2018;14(6):1534-1538.

39. Biggart R, Finn A, Marlow R. Lack of impact of rotavirus vaccination on childhood seizure hospitalizations in England - an interrupted time series analysis. Vaccine. 2018;36(31):4589-4592.

40. Vaarala O, Jokinen J, Lahdenkari M, Leino T. Rotavirus vaccination and the risk of celiac disease or type 1 diabetes in Finnish children at early life. Pediatr Infect Dis J. 2017;36(7):674-675.

41. Arias CF, Romero P, Álvarez V, López S. Trypsin activation pathway of rotavirus infectivity. J Virol. 1996;70(9):5832-5839.

42. Rodríguez JM, Chichón FJ, Martín-Forero E, et al. New insights into rotavirus entry machinery: stabilization of rotavirus spike conformation is independent of trypsin cleavage. PLoS Pathog. 2014;10(5):e1004157.

43. Hu L, Crawford SE, Czako R, et al. Cell attachment protein VP8* of a human rotavirus specifically interacts with A-type histo-blood group antigen. Nature. 2012;485(7397):256-259.

44. Mossel EC, Ramig RF. A lymphatic mechanism of rotavirus extraintestinal spread in the neonatal mouse. JVirol. 2003;77(22):12352-12356.

45. Weclewicz K, Svensson L, Kristensson K. Targeting of endoplasmic reticulum-associated proteins to axons and dendrites in rotavirusinfected neurons. Brain Res Bull. 1998;46(4):353-360.

46. Salas A, Marco-Puche G, Triviño JC, et al. Strong down-regulation of glycophorin genes: a host defense mechanism against rotavirus infection. Infect Genet Evol. 2016;44:403-411.

47. Isa P, Arias CF, López S. Role of sialic acids in rotavirus infection. Glycoconj J. 2006;23(1-2):27-37.

48. Jiang X, Liu Y, Tan M. Histo-blood group antigens as receptors for rotavirus, new understanding on rotavirus epidemiology and vaccine strategy. Emerg Microbes Infect. 2017;6(4):e22.

49. Nordgren J, Sharma S, Bucardo F, et al. Both Lewis and secretor status mediate susceptibility to rotavirus infections in a rotavirus genotypedependent manner. Clin Infect Dis. 2014;59(11):1567-1573.

50. Bustamante M, Standl M, Bassat Q, et al. A genome-wide association meta-analysis of diarrhoeal disease in young children identifies FUT2 locus and provides plausible biological pathways. Hum Mol Genet. 2016;25(18):4127-4142.

51. Imbert-Marcille BM, Barbé L, Dupé M, et al. A FUT2 gene common polymorphism determines resistance to rotavirus A of the $\mathrm{P}[8]$ genotype J Infect Dis. 2014;209(8):1227-1230. 
52. Bucardo F, Nordgren J, Reyes Y, González F, Sharma S, Svensson L. The Lewis A phenotype is a restriction factor for Rotateq and Rotarix vaccine-take in Nicaraguan children. Sci Rep. 2018;8(1):1502.

53. Ferrer-Admetlla A, Sikora M, Laayouni H, et al. A natural history of FUT2 polymorphism in humans. Mol Biol Evol. 2009;26(9) :1993-2003.

54. Kazi AM, Cortese MM, Yu Y, et al. Secretor and salivary ABO blood group antigen status predict rotavirus vaccine take in infants. $J$ Infect Dis. 2017;215(5):786-789.

55. Iturriza-Gómara $\mathrm{M}$, Dallman $\mathrm{T}$, Bányai $\mathrm{K}$, et al. Rotavirus genotypes co-circulating in Europe between 2006 and 2009 as determined by EuroRotaNet, a pan-European collaborative strain surveillance network. Epidemiol Infect. 2011;139(6):895-909.

56. Gale P, Hill A, Kelly L, et al. Applications of omics approaches to the development of microbiological risk assessment using RNA virus dose-response models as a case study. J Appl Microbiol. 2014;117(6):1537-1548.

57. Uchiyama R, Chassaing B, Zhang B, Gewirtz AT. Antibiotic treatment suppresses rotavirus infection and enhances specific humoral immunity. J Infect Dis. 2014;210(2):171-182.

58. Freitas M, Axelsson LG, Cayuela C, Midtvedt T, Trugnan G. Indigenous microbes and their soluble factors differentially modulate intestinal glycosylation steps in vivo. Use of a "lectin assay" to survey in vivo glycosylation changes. Histochem Cell Biol. 2005;124(5):423-433.

59. Parker EPK, Praharaj I, Zekavati A, et al. Influence of the intestinal microbiota on the immunogenicity of oral rotavirus vaccine given to infants in south India. Vaccine. 2018;36(2):264-272.

60. García-López R, Pérez-Brocal V, Diez-Domingo J, Moya A. Gut microbiota in children vaccinated with rotavirus vaccine. Pediatr Infect Dis J. 2012;31(12):1300-1302.

61. Broquet AH, Hirata Y, McAllister CS, Kagnoff MF. RIG-I/MDA5/ MAVS are required to signal a protective IFN response in rotavirusinfected intestinal epithelium. J Immunol. 2011;186(3):1618-1626.

62. Lin JD, Feng N, Sen A, et al. Distinct roles of type I and type III interferons in intestinal immunity to homologous and heterologous rotavirus infections. PLoS Pathog. 2016;12(4):e1005600.
63. López S, Sánchez-Tacuba L, Moreno J, Arias CF. Rotavirus strategies against the innate antiviral system. Annu Rev Virol. 2016;3(1): 591-609.

64. Barro M, Patton JT. Rotavirus nonstructural protein 1 subverts innate immune response by inducing degradation of IFN regulatory factor 3 . Proc Natl Acad Sci U S A. 2005;102(11):4114-4119.

65. Holloway G, Dang VT, Jans DA, Coulson BS. Rotavirus inhibits IFNinduced STAT nuclear translocation by a mechanism that acts after STAT binding to importin- $\alpha$. J Gen Virol. 2014;95(Pt 8):1723-1733.

66. Zhu S, Ding S, Wang P, et al. Nlrp9b inflammasome restricts rotavirus infection in intestinal epithelial cells. Nature. 2017;546(7660):667-670.

67. Zhang B, Chassaing B, Shi Z, et al. Viral infection. Prevention and cure of rotavirus infection via TLR5/NLRC4-mediated production of IL-22 and IL-18. Science. 2014;346(6211):861-865.

68. Mesa MC, Gutiérrez L, Duarte-Rey C, Angel J, Franco MA. A TGF-beta mediated regulatory mechanism modulates the $\mathrm{T}$ cell immune response to rotavirus in adults but not in children. Virology. 2010;399(1):77-86.

69. Franco MA, Greenberg HB. Role of B cells and cytotoxic T lymphocytes in clearance of and immunity to rotavirus infection in mice. $J$ Virol. 1995;69(12):7800-7806.

70. Egli A, Santer DM, O'Shea D, Tyrrell DL, Houghton M. The impact of the interferon-lambda family on the innate and adaptive immune response to viral infections. Emerg Microbes Infect. 2014;3(7):e51.

71. Syedbasha M, Egli A. Interferon lambda: modulating immunity in infectious diseases. Front Immunol. 2017;8:119.

72. Sheridan SL, Ware RS, Grimwood K, Lambert SB. Febrile Seizures in the Era of Rotavirus Vaccine. J Pediatric Infect Dis Soc. 2016;(5)2:206-209.

73. Pringle KD, Burke RM, Steiner CA, Parashar UD, Tate JE. Trends in Rate of Seizure-Associated Hospitalizations Among Children $<5$ Years Old Before and After Rotavirus Vaccine Introduction in the United States, 2000-2013. J Infect Dis. 2018;217(4):581-588.

73. Burke RM, Tate JE, Dahl RM, Aliabadi N, Parashar UD. Rotavirus Vaccination Is Associated with Reduced Seizure Hospitalization Risk Among Commerciallly Insured U.S. Children. Clin Infect Dis. 2018;67(10):1614-1616.
Infection and Drug Resistance

\section{Publish your work in this journal}

Infection and Drug Resistance is an international, peer-reviewed openaccess journal that focuses on the optimal treatment of infection (bacterial, fungal and viral) and the development and institution of preventive strategies to minimize the development and spread of resistance. The journal is specifically concerned with the epidemiology of antibiotic

\section{Dovepress}

resistance and the mechanisms of resistance development and diffusion in both hospitals and the community. The manuscript management system is completely online and includes a very quick and fair peerreview system, which is all easy to use. Visit http://www.dovepress.com/ testimonials.php to read real quotes from published authors. 